\title{
Lattice-Gas Automata for the Navier-Stokes Equation
}

\author{
U. Frisch \\ Centre National de la Recherche Scientifique, Observatoire de Nice, 06003 Nice Cedex, France
}

B. Hasslacher

Theoretical Division and Center for Nonlinear Studies, Los Alamos National Laboratory, Los Alamos, New Mexico 87545

and

Y. Pomeau

Centre National de la Recherche Scientifique, Ecole Normale Supérieure, 75231 Paris Cedex, France, and Service de Physique Théorique, Centre d'Etudes Nucléaires de Saclay, 91191 Gif-sur-Yvette, France

(Received 22 October 1985)

\begin{abstract}
We show that a class of deterministic lattice gases with discrete Boolean elements simulates the Navier-Stokes equation, and can be used to design simple, massively parallel computing machines.
\end{abstract}

PACS numbers: $89.80 .+\mathrm{h}$

The relatively recent availability of sophisticated interactive digital simulation has led to considerable progress in the unraveling of universal features of complexity generated by nonlinear dynamical systems with few degrees of freedom. In contrast, nonlinear systems with many degrees of freedom, e.g., highReynolds-number flow, are understood only on a quite superficial level, ${ }^{1}$ and are likely to remain so, unless they can be explored in depth, e.g., by interactive simulation. This is many orders of magnitude beyond the capacity of existing computational resources. There are similar limitations on our ability to simulate many other multidimensional field theories.

Massively parallel architectures and algorithms are needed to avoid the ultimate computation limits of the speed of light and various solid-state constraints. Also, when parameter space must be explored quickly and extreme accuracy is unnecessary, a floating-point representation may not be efficient. For example, to compute the drag due to turbulent flow past an obstacle with a modest accuracy of 5 bits, common experience in computational fluid dynamics shows that intermediate computations require from 32 to 64 bits. Floating-point representations hierarchically favor bits in the most significant places, ${ }^{2}$ which is a major cause of numerical instability. In principle, schemes which give bits equal weight would be preferable. Because of roundoff noise, a floating-point calculation can run away to unphysical regimes, in an attempt to treat each bit equally.

A simulation strategy can be devised which both is naturally parallel and treats all bits on an equal footing, for systems which evolve by discrete cellular automaton rules, with only local interactions. ${ }^{3}$ This avoids the complex switching networks which limit the computational power of conventional parallel arrays.

There has been speculation that various physically interesting field equations can be approximated by the large-scale behavior of suitably chosen cellular automata. $^{4}$ We shall here construct lattice-gas automata which asymptotically go over to the incompressible $2 \mathrm{D}$ and 3D Navier-Stokes equations.

To understand the physics behind lattice gases, we first point out that a fluid can be described on three levels: the molecular level at which motion, usually Hamiltonian, is reversible; the kinetic level, in the irreversible low-density Boltzmann approximation; and the macroscopic level, in the continuum approximation. At the first two levels of description, the fluid is near thermodynamic equilibrium. In the last there are free thermodynamic variables: local density, momentum, temperature, etc. A macroscopic description of the fluid comes about by a patching together of equilibria which are varying slowly in space and time, implying continuum equations for thermodynamic variables as consistency conditions. This was first realized by Maxwell, ${ }^{5}$ and put in final form by Chapman and Enskog. ${ }^{6}$

There are many ways of building microscopic models that lead to a given set of continuum equations. It is known that one can build two- and threedimensional Boltzmann models, with a small number of velocity vectors, which, in the continuum limit, reproduce quite accurately major fluid dynamical features (e.g., shock waves in a dilute gas, etc. ${ }^{7}$ ). Such Boltzmann models are fundamentally probabilistic, discrete only in velocity, but continuous in space and time. In contrast, we will use lattice-gas models, which have a completely discrete phase space and time and therefore may be viewed as made of "Boolean molecules."

The simplest case is the Hardy, de Pazzis, and Pomeau model $^{8}$ (hereafter called HPP) which has an underlying regular, square, two-dimensional lattice 
with unit link lengths. At each vertex, there are up to four molecules of equal mass, with unit speed, whose velocities point in one of the four link directions. The simultaneous occupation of a vertex by identical molecules is forbidden. Time is also discrete. The update is as follows. First, each molecule moves one link, to the nearest vertex to which its velocity was pointing. Then, any configuration of exactly two molecules moving in opposite directions at a vertex (head-on collisions) is replaced by another one at right angles to the original. All other configurations are left unchanged. The HPP model has a number of important properties. ${ }^{8}$ The crucial one is the existence of thermodynamic equilibria. No ergodic theorem is known, but relaxation to equilibrium has been demonstrated numerically. ${ }^{8}$ These equilibria have free continuous parameters, namely, the average density and momentum. The equilibrium distribution functions are completely factorized over vertices and directions, being independent of vertex position, but dependent on direction, unless the mean momentum vanishes. When density and momentum are varied slowly in space and time, "macrodynamical" equations emerge which differ from the nonlinear Navier-Stokes equations in three respects.

The discrepancies may be classified as (1) lack of Galilean invariance, (2) lack of isotropy, and (3) a crossover dimension problem. Galilean invariance is by definition broken by the lattice; consequently, thermodynamic equilibria with different velocities cannot be related by a simple transformation. This is reflected by the nonlinear term in the momentum equation, containing a momentum flux tensor, which not only has quadratic terms in the hydrodynamic velocity $\mathbf{u}$, as it should be in the Navier-Stokes equation, but also has nonlinear corrections to arbitrarily high order in the velocity. However, these terms are negligible at low Mach number, a condition which also guarantees incompressibility. The HPP automaton is invariant under $\pi / 2$ rotations. Such a lattice symmetry is insufficient to insure the isotropy of the fourth degree tensor relating momentum flux to quadratic terms in the velocity. Finally, crossover dimension is a general property of two-dimensional hydrodynamics, when thermal noise is added to the Navier-Stokes equations or to the HPP version of it. Simply put, the viscosity develops a logarithmic scale dependence, which is a dimensional crossover phenomenon, common in phase transitions and field theory. ${ }^{9}$ In three dimensions, this difficulty does not exist.

Focusing on the isotropy problem, we note that for the HPP model, the momentum flux tensor has the form

$$
P_{\alpha \beta}=p \delta_{\alpha \beta}+T_{\alpha \beta \gamma \epsilon} u_{\gamma} u_{\epsilon}+O\left(u^{4}\right) .
$$

Here $p=\rho / 2$ is the pressure; terms odd in $\mathbf{u}$ vanish by parity. The tensor $T$ is, by construction, pairwise symmetric in both $(\alpha, \beta)$ and $(\gamma, \epsilon)$. Observe that when the underlying microworld is two-dimensional and invariant under the hexagonal rotation group (multiples of $\pi / 3)$, the tensor $T$ is isotropic and (1) takes the form

$$
P_{\alpha \beta}=\left(p+\mu u^{2}\right) \delta_{\alpha \beta}+\lambda u_{\alpha} u_{\beta}+O\left(u^{4}\right),
$$

with suitable scalar factors $\lambda$ and $\mu$. At low Mach number this is the correct form for the Navier-Stokes equation. This observation appears to be new. So, in two dimensions, we will use a triangular instead of a square lattice. Each vertex then has a hexagonal neighborhood (Fig. 1). We will call this model the hexagonal lattice gas (HLG). The setup is the same as in the HPP lattice gas, except for modified collision rules. A suitable set is one given by Harris, ${ }^{10}$ in connection with a discrete Boltzmann model, supplemented by a Fermi exclusion condition, of single occupation of each Boolean state. The Fermi-modified Harris rules are as follows: Number the six links out of any vertex counterclockwise, with an index $i$, defined on the integers (mod6). There are both two- and threebody collisions. For two-body collisions, we have $(i, i+3)$ goes to (a) $(i+1, i-2)$ or (b) $(i-1, i+2)$. Type $\mathrm{a}$ and $\mathrm{b}$ outcomes have equal $a$ priori weights. For three-body collisions we have $(i, i+2, i-2)$ goes to $(i+3, i+1, i-1)$. In these rules, it is assumed that no incident link to a vertex is populated, other than the ones given as initial states. All other configurations remain unaffected by collisions. These rules are designed to conserve particle number and momentum at each vertex, i.e., a total of three scalar conservation relations. Without three-body collisions, there would be four scalar conservation relations, namely mass and

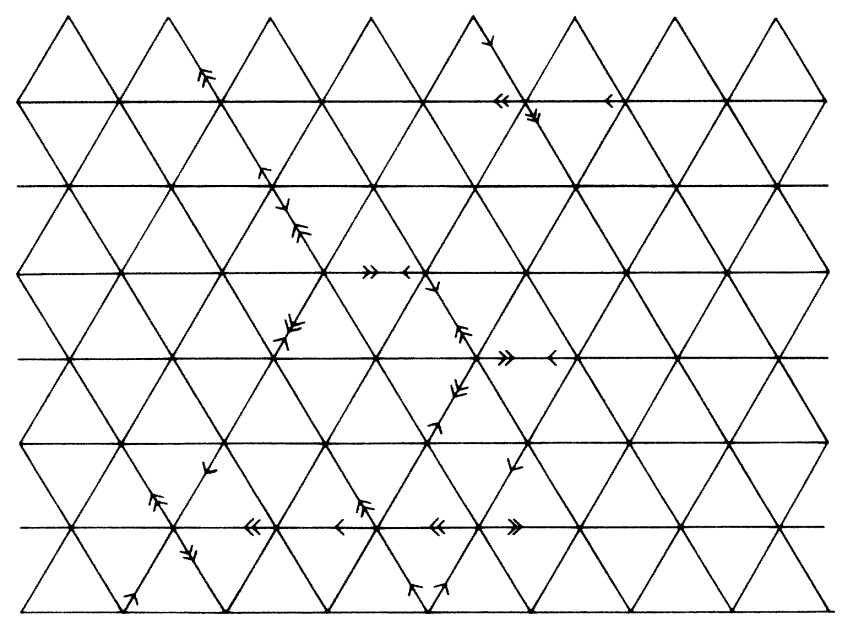

FIG. 1. Triangular lattice with hexagonal symmetry and hexagonal lattice-gas rules. Particles at time $t$ and $t+1$ are marked by single and double arrows, respectively. 
momentum along each of the three lattice directions.

Note that the HPP rules are invariant under duality (interchange of particles and holes), whereas the present rules are not. Duality can be restored by addition of suitable four-particle collision rules, but we will not use them here.

We display a variant of this model where at most one particle is allowed to remain at rest at each vertex. The rest particles are labeled by an asterisk and the previous rules are supplemented with $(i, i+2)$ goes to $\left(i-2,,^{*}\right)$ and $(i, *)$ goes to $(i+2, i-2)$. Additional variations on the model allow one to define a nontrivial temperature. The remainder of this discussion is concerned only with the basic (HLG) model.

We briefly outline how the hexagonal lattice gas leads to the two-dimensional Navier-Stokes equations. A detailed derivation will be presented elsewhere. ${ }^{11}$ Let $N_{i}$ be the average population at a vertex with velocity in the direction $i$. The average is over a macroscopic space-time region so that $N_{i}$ depends slowly on space and time variables. We define a slowly varying density $\rho$ and momentum $\rho u$ by

$$
\rho=\Sigma_{i} N_{i}, \quad \rho \mathrm{u}=\sum_{i} N_{i} \mathrm{c}_{i},
$$

where $\mathbf{c}_{i}$ is a unit vector in the direction $i$. Locally, for a given $\rho$ and $\mathbf{u}$, the $N_{i}$ 's can be computed from both these definitions and the detailed-balance equations at thermodynamic equilibrium, which are too involved to present here. This gives a Fermi-Dirac distribution:

$$
N_{i}=\left\{1+\exp \left[\alpha(\rho, u)+\beta(\rho, u) \mathbf{c}_{i} \cdot \mathbf{u}\right]\right\}^{-1} .
$$

In general, $\alpha$ and $\beta$ satisfy equations with no simple solutions. However, when $\mathbf{u}=0$, it is obvious by symmetry that $N_{i}=\rho / 6$. Therefore, $\alpha$ and $\beta$ can be expanded in a Taylor series around $\mathbf{u}=0$. The result can be used to compute mass and momentum flux to first order in the macroscopic gradients. Second-order terms in the gradients (viscous terms) are obtained by Green-Kubo relations or by a Chapman-Enskog expansion. ${ }^{12}$ The following set of hydrodynamic equations is thus obtained:

$$
\begin{aligned}
& \partial \rho / \partial t+\nabla \cdot(\rho \mathbf{u})=0, \\
& \frac{\partial}{\partial t}\left(\rho u_{\alpha}\right)+\sum_{\boldsymbol{\beta}} \frac{\partial}{\partial x_{\beta}}\left[g(\rho) \rho u_{\alpha} u_{\beta}+O\left(u^{4}\right)\right] \\
& \quad=-\frac{\partial}{\partial x_{\alpha}} p+\eta_{1}(\rho) \nabla^{2} u_{\alpha}+\eta_{2}(\rho) \frac{\partial}{\partial x_{\alpha}} \nabla \cdot \mathbf{u},
\end{aligned}
$$

with $g(\rho)=(\rho-3) /(\rho-6)$ and $p=\rho / 2 . \eta_{1}(\rho)$ and $\eta_{2}(\rho)$ are the shear and bulk viscosities. ${ }^{12}$

Deletion of the nonlinear and viscous terms gives the wave equation for sound waves propagating isotropically with a speed equal to the "velocity of light" (here set equal to 1) over $\sqrt{2}$, just as for a twodimensional photon gas. These sound waves have been observed in simulations on the MIT cellular automaton machine by Margolus, Toffoli, and Vichniac. ${ }^{13}$ They used lattice-gas models that yield the same wave equation as above.

The nonlinear system (5) and (6) goes over to the incompressible Navier-Stokes equation by the following limiting procedure: Let the Mach number $M$ $=u \sqrt{2}$ tend to zero, and the hydrodynamic scale $L$ tend to infinity, while keeping their product fixed. As in the usual derivation of the incompressible limit, density fluctuations become irrelevant, except in the pressure term; also, the continuity equation (5) reduces to $\nabla \cdot \mathbf{u}=0$. Thus, the factor $g(\rho)$ is to leading order a constant and may, for $0<\rho<3$, be absorbed in a rescaled time. The resulting Reynolds number is

$$
N_{\mathrm{R}}=M L \rho g(\rho) / \sqrt{2} \eta_{1}(\rho) .
$$

Note that Galilean invariance, which does not hold at the lattice level, is restored macroscopically.

A straightforward lift of the hexagonal lattice-gas model from two into three dimensions does not work. The reason is that the regular space-filling simplex with the greatest symmetry in three dimensions is the face centered cubic, with twelve equal-speed velocity directions out of each vertex. Unfortunately, the relevant tensors such as $T_{\alpha \beta \gamma \epsilon}$ in Eq. (1) depend now on three constants. This induces a spurious, isotropybreaking term in the Navier-Stokes equations, proportional to $\left(\partial / \partial x_{\alpha}\right) u_{\alpha}^{2}$ (no summation on $\alpha$ ).

This obstacle may be removed by a splitting method. The nonlinear term in the three-dimensional NavierStokes equation is recast as the sum of two terms, each containing spurious elements and each realizable on a different lattice (for example, a face-centered-cubic lattice and a regular cubic lattice).

In lattice-gas models, as in general cellular automata (CA's), boundary conditions are very easy to implement. Specular reflection of molecules gives so-called "free slip" boundary conditions for the hydrodynamic velocity u. "Rigid" boundary conditions are obtained either by random scattering of particles back into the incoming half plane from a locally planar boundary, or by specular reflection from a microscale roughened version of the macroscopic boundary.

We mention some practical limitations on lattice-gas models. For the hydrodynamic description to hold, there must be a scale separation between the smallest hydrodynamic scale and the lattice link length; as we shall see, this requirement is automatically satisfied. Lattice-gas models must be run at moderate Mach numbers $M$ (say 0.3 to 0.5 ), to remain incompressible, and to avoid spurious high-order nonlinear terms. For fixed Mach number, the largest Reynolds number associated with a $D$-dimensional lattice with $O(N)$ sites in each direction is $O(N)$. This is because in our 
units, the kinematic viscosity of the hexagonal lattice gas is $O(1)$. From standard turbulence theory, ${ }^{14}$ it follows that the dissipation scale is $O\left(N^{1 / 2}\right)$ in $2 \mathrm{D}$ and $O\left(N^{1 / 4}\right)$ in 3D. This insures the required scale separation at large Reynolds numbers. It would, however, be desirable to reduce the scale separation, especially in $2 \mathrm{D}$, to avoid excessive storage requirements compared to conventional incompressible floatingpoint simulations (in the latter, the mesh can be taken comparable to the dissipation scale).

For this, we observe that the viscosity in the lattice gas is decreased by a factor $P$ if we subdivide each cell into a sublattice with links $P$ times smaller. We note also that the sublattice need not be similar to the original lattice. It must have the same collision rules, to preserve local thermodynamic equilibria, but the geometry does not matter since macroscopic quantities may be considered uniform over the cell. Thus, all the sublattice vertices in a given cell may be regarded as indistinguishable and can be coded in $O(\ln P)$ rather than $O\left(P^{D}\right)$ bits; interactions occur between randomly chosen vertex pairs within cells and between neighboring cells; the latter being less frequent by a factor $O(1 / P)$.

Simulations of the models discussed here, done on general-purpose computers and exhibiting a variety of known two-dimensional hydrodynamic phenomena, have been made by d'Humières, Lallemand, and Shimomura. ${ }^{15}$

We have given a concrete hydrodynamical example of how CA's can be used to simulate classical nonlinear fields. We expect that further CA implementations will be found for the Navier-Stokes equation and other problems, not necessarily based on thermalized lattice gases and possibly less constrained than ours.

S. Wolfram stimulated our interest in cellular automata as a possible new approach to turbulence phenomena. Acknowledgments are also due to T. Bloch, R. Caflish, D. d'Humières, R. Gatignol, R. Kraichnan, P. Lallemand, N. Margolus, D. Nelson, J. L. Oneto, S. A. Orszag, J. P. Rivet, T. Shimomura, Z. S. She, B. Shraiman, T. Toffoli, and G. Vichniac, as well as the following: Woods Hole Geophysical Fluid Dynamics Summer Program (U.F., Y.P.); Aspen Center for Physics, 1985 Chaos Workshop (B.H.); and
Service de Physique Théorique, Centre d'Etudes Nucleaires de Saclay (B.H.). This work was supported in part by National Science Foundation Grant No. 8442384.

1U. Frisch, Phys. Scr. T9, 131 (1985).

${ }^{2}$ D. E. Knuth, The Art of Computer Programming: Semi Numerical Algorithms (Addison-Wesley, Reading, Mass., 1981), Vol. 2, p. 238.

${ }^{3}$ T. Toffoli, Physica (Amsterdam) 10D, 117 (1984); S. Wolfram, Nature 311, 419 (1984).

${ }^{4}$ Y. Pomeau, J. Phys. A 17, L415 (1984); G. Vichniac, Physica (Amsterdam) 10D, 96 (1984), and references therein; N. Margolus, Physica (Amsterdam) 10D, 81 (1984).

5J. C. Maxwell, The Scientific Papers, Vol. 2 (Cambridge Univ. Press, Cambridge, England, 1890), p. 681.

${ }^{6}$ G. E. Uhlenbeck and G. W. Ford, Lectures in Statistical Mechanics, Lectures in Applied Math Vol. 1 (American Mathematical Society, Providence, R.I., 1963).

7J. E. Broadwell, Phys. Fluids 7, 1243 (1964); R. Gatignol, Théorie Cinétique des Gaz à Répartition Discrète des Vitesses, Lecture Notes in Physics Vol. 36 (Springer, Berlin, 1975).

8J. Hardy and Y. Pomeau, J. Math. Phys. 13, 1042 (1972); J. Hardy, Y. Pomeau, and O. de Pazzis, J. Math. Phys. 14, 1746 (1973); J. Hardy, O. de Pazzis, and Y. Pomeau, Phys. Rev. A 13, 1949 (1976).

${ }^{9}$ D. Forster, D. R. Nelson, and M. J. Stephen, Phys. Rev. A 16, 732 (1977).

${ }_{10}$ S. Harris, Phys. Fluids 9, 1328 (1966).

${ }^{11} \mathrm{U}$. Frisch, B. Hasslacher, and Y. Pomeau, "Hydrodynamics on Lattice Gases," to be published.

12J. Rivet and U. Frisch, C.R. Seances Acad. Sci., Ser. 2 302, 267 (1986).

${ }^{13}$ N. Margolus, T. Toffoli, and G. Vichniac, private communication, and Massachusetts Institute of Technology Technical Memo No. LCS-TM-296, 1984 (unpublished).

${ }^{14}$ A. N. Kolmogorov, C.R. (Dokl.) Acad. Sci. USSR 30, 301, 538 (1941); R. Kraichnan, Phys. Fluids 10, 1417 (1967); G. K. Batchelor, Phys. Fluids 12, Suppl. 2, 233 (1969).

${ }^{15} \mathrm{D}$. d'Humières, P. Lallemand, and T. Shimomura, "Lattice gas cellular automata, a new experimental tool for hydrodynamics," to be published. 